\title{
Study on Nonlinear Phenomena in Buck-Boost Converter with Switched-Inductor Structure
}

\author{
Hongchen Liu and Shuang Yang \\ School of Electrical Engineering and Automation, Harbin Institute of Technology, Harbin 150001, China \\ Correspondence should be addressed to Hongchen Liu; fenmiao@hit.edu.cn
}

Received 8 May 2013; Accepted 1 October 2013

Academic Editor: Moez Feki

Copyright (C) 2013 H. Liu and S. Yang. This is an open access article distributed under the Creative Commons Attribution License, which permits unrestricted use, distribution, and reproduction in any medium, provided the original work is properly cited.

The switched-inductor structure can be inserted into a traditional Buck-Boost converter to get a high voltage conversion ratio. Nonlinear phenomena may occur in this new converter, which might well lead the system to be unstable. In this paper, a discrete iterated mapping model is established when the new Buck-Boost converter is working at continuous conduction current-controlled mode. On the basis of the discrete model, the bifurcation diagrams and Poincare sections are drawn and then used to analyze the effects of the circuit parameters on the performances. It can be seen clearly that various kinds of nonlinear phenomena are easy to occur in this new converter, including period-doubling bifurcation, border collision bifurcation, tangent bifurcation, and intermittent chaos. Value range of the circuit parameters that may cause bifurcations and chaos are also discussed. Finally, the timedomain waveforms, phase portraits, and power spectrum are obtained by using Matlab/Simulink, which validates the theoretical analysis results.

\section{Introduction}

In recent years, much attention was paid to the switchedinductor structure because of its several unique characteristics. One of the most remarkable feature of the switchedinductor structure is that it can be combined with the traditional DC-DC converters to provide new converters with a steep voltage conversion ratio $[1,2]$. The DC-DC converter is strongly nonlinear system, and it has rich irregular phenomena like subharmonic oscillations, time-bifurcation, quasi-periodicity, chaos, and so on [3-8].

The nonlinear phenomena in the new Buck-Boost converter may be rather complex than that in the traditional converters, which will deteriorate the performance of the converter to some extent. The bifurcation and the chaos in traditional DC-DC converters were studied extensively [914]. It was demonstrated that there was various kinds of nonlinear phenomena in the traditional Buck-Boost converter [15-17]. However, very little attention was paid to complex behaviors in Buck-Boost converter combined with the switched-inductor structure.

For this reason, the nonlinear phenomena in a currentmode controlled Buck-Boost converter with switchedinductor structure are studied in this paper. The discrete iterated mapping model under continuous conduction mode (CCM) is established. The bifurcation diagrams and Poincare sections are drawn based on the discrete-time model, which intuitively reflect the influence of the circuit parameters on the system performance. It can be shown clearly that many kinds of nonlinear phenomena existing in this new converter, such as period-doubling bifurcation, border collision bifurcation, tangent bifurcation, and intermittent chaos. At last, the time-domain waveform, phase portraits, and power spectrum under various load resistance are obtained by Matlab/Simulink, which validates the conclusions that came from the theoretical analysis. The research result provides an important reference for engineering design and performance analysis.

\section{Operation Principle and Modeling of the Converter}

2.1. Operation Principle. The schematic of the new currentmode controlled Buck-Boost converter with switchedinductor structure is shown in Figure 1. In general, the value of $L_{1}$ equals $L_{2}$ in this $L$-switching structure according to [1], and all elements are assumed to be ideal ones. Let current 


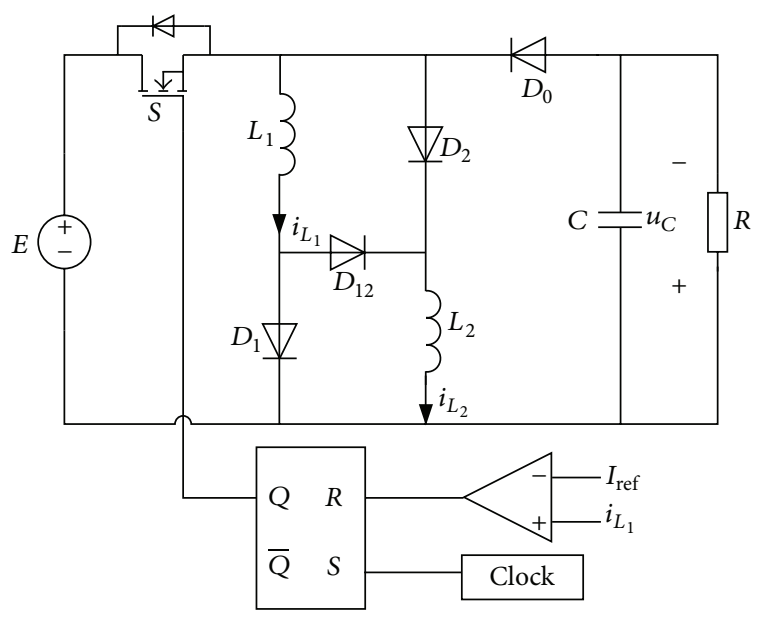

FIGURE 1: Current-mode controlled buck-boost converter with switched-inductor structure.

flowing through $L_{1}$ (i.e., $i_{L_{1}}$ ) be the control signal, operation process of this system is described as follows. A feedback path composed of an RS trigger and a comparator was used to control switch $S$. In the comparator, the current $i_{L_{1}}$ is compared with a reference current $I_{\text {ref }}$ to generate on-off signal. At the beginning of a cycle, switch $S$ is turned on. It remains on state so that the $i_{L_{1}}$ increases, until it reaches the value $I_{\text {ref }}$. Then, the switch $S$ is turned off, and it remains off state until the next cycle begins. Thus, the converter toggles between two linear configurations when it operates in CCM. The switching topologies are shown in Figure 2.

As shown in Figure 2, $L_{1}$ and $L_{2}$ are being charged in parallel when the switch is on; while they are being discharged in series when the switch is off. Two parameters are defined as follows: $L_{\mathrm{eq}}$ is denoted as the equivalent inductor of $L_{1}$ and $L_{2}$, and $i_{\text {eq }}$ is denoted as the current that flows through the $L_{\text {eq }}$. And an assumption is made that the switch $S$ is on during $\left[t_{0}, t_{1}\right]$ and is off during $\left[t_{1}, t_{2}\right]$. Therefore, both of these two configurations can be characterized by a dynamic matrix and an input matrix, say $A_{1}, A_{2}$ and $B_{1}, B_{2}$, respectively.

Let $x=\left[\begin{array}{ll}i_{\text {eq }} & u_{C}\end{array}\right]^{T}$ be a vector of state variables, the differential equation of the configuration can be written as follows during $\left[t_{0}, t_{1}\right]$ :

$$
\dot{x}=A_{1} x+B_{1} E, \quad t_{0} \leq t \leq t_{1},
$$

where

$$
A_{1}=\left[\begin{array}{cc}
0 & 0 \\
0 & -\frac{1}{R C}
\end{array}\right], \quad B_{1}=\left[\begin{array}{c}
1 \\
L_{\mathrm{eq}} \\
0
\end{array}\right] .
$$

Similarly, the differential equation can be written as follows during $\left[t_{1}, t_{2}\right]$ :

$$
\dot{x}=A_{2} x+B_{2} E, \quad t_{1} \leq t \leq t_{2},
$$

where

$$
A_{2}=\left[\begin{array}{cc}
0 & -\frac{1}{L_{\mathrm{eq}}} \\
\frac{1}{C} & -\frac{1}{R C}
\end{array}\right], \quad B_{2}=\left[\begin{array}{l}
0 \\
0
\end{array}\right] .
$$

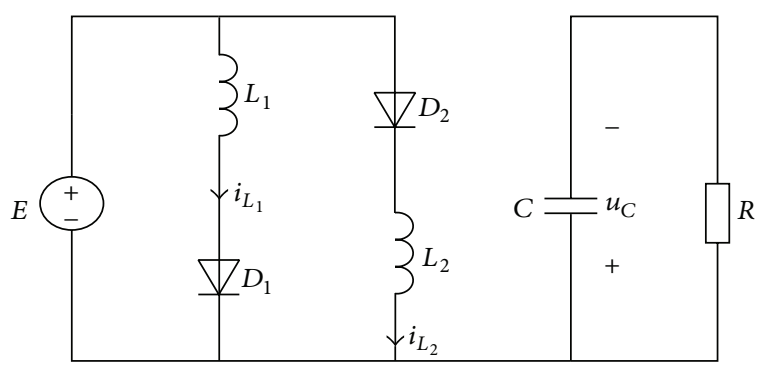

(a)

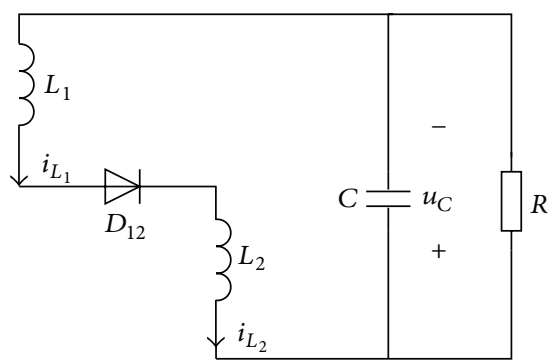

(b)

FIGURE 2: Switching topologies of the converter. (a) Switch is on; (b) switch is off.

Noting that there is a conversion between $L_{1}$ and $L_{2}$ from parallel connection to series connection, the value of $L_{\text {eq }}$ and $i_{\mathrm{eq}}$ in (1) and (3) are not equal. Taking $L_{1}=L_{2}$ into consideration, we can get $L_{\mathrm{eq}}$ and $i_{\mathrm{eq}}$ in (1) as follows:

$$
L_{\text {eq }}=\frac{L_{1} \times L_{2}}{L_{1}+L_{2}}=\frac{1}{2} L_{1}, \quad i_{\mathrm{eq}}=i_{L_{1}}+i_{L_{2}}=2 i_{L_{1}} .
$$

Also, we can get $L_{\mathrm{eq}}$ and $i_{\mathrm{eq}}$ in (3) as follows:

$$
L_{\text {eq }}=L_{1}+L_{2}=2 L_{1}, \quad i_{\text {eq }}=i_{L_{1}}=i_{L_{2}} .
$$

Considering that $i_{L_{1}}$ is the control signal, we redefine $x^{\prime}=\left[\begin{array}{ll}i_{L_{1}} & u_{C}\end{array}\right]^{T}$ as a new vector in order to simplify the derivation of the discrete iterated mapping model. And the corresponding differential equations can be obtained according to (1)-(6), as shown by

$$
\begin{array}{ll}
\dot{x}^{\prime}=A_{1}^{\prime} x^{\prime}+B_{1}^{\prime} E, & t_{0} \leq t \leq t_{1}, \\
\dot{x}^{\prime}=A_{2}^{\prime} x^{\prime}+B_{2}^{\prime} E, & t_{1} \leq t \leq t_{2},
\end{array}
$$

where $A_{1}^{\prime}=\left[\begin{array}{cc}0 & 0 \\ 0 & -1 / R C\end{array}\right], A_{2}^{\prime}=\left[\begin{array}{cc}0 & -1 /\left(2 L_{1}\right) \\ 1 / C & -1 / R C\end{array}\right], B_{1}^{\prime}=\left[\begin{array}{c}1 / L_{1} \\ 0\end{array}\right]$, and $B_{2}^{\prime}=\left[\begin{array}{l}0 \\ 0\end{array}\right]$.

According to the control theory, solutions of (7) and (8) can be written as

$$
\begin{aligned}
x^{\prime}\left(t_{1}\right) & =f_{1}\left(x^{\prime}\left(t_{0}\right), t_{1}\right) \\
& =e^{A_{1}^{\prime}\left(t_{1}-t_{0}\right)}\left[x^{\prime}\left(t_{0}\right)+\int_{t_{0}}^{t_{1}} e^{A_{1}^{\prime}\left(t_{0}-\tau\right)} B_{1}^{\prime} E d \tau\right] \\
& =\phi_{1}\left(t_{1}-t_{0}\right)\left[x^{\prime}\left(t_{0}\right)+\int_{t_{0}}^{t_{1}} \phi_{1}\left(t_{0}-\tau\right) B_{1}^{\prime} E d \tau\right],
\end{aligned}
$$




$$
\begin{aligned}
x^{\prime}\left(t_{2}\right) & =f_{2}\left(x^{\prime}\left(t_{1}\right), t_{2}\right) \\
& =e^{A_{2}^{\prime}\left(t_{2}-t_{1}\right)}\left[x^{\prime}\left(t_{1}\right)+\int_{t_{1}}^{t_{2}} e^{A_{2}^{\prime}\left(t_{1}-\tau\right)} B_{2}^{\prime} E d \tau\right] \\
& =\phi_{2}\left(t_{2}-t_{1}\right)\left[x^{\prime}\left(t_{1}\right)+\int_{t_{1}}^{t_{2}} \phi_{2}\left(t_{1}-\tau\right) B_{2}^{\prime} E d \tau\right],
\end{aligned}
$$

where $\phi_{i}(t)=e^{A_{i}^{\prime} t}, i=1,2 ; \phi_{i}(t)$ is state transition matrix of $A_{i}^{\prime}$.

2.2. Discrete Iterated Mapping Model. In general, the discrete time model can be established by means of stroboscopic map, S-switching map, or A-switching map. The stroboscopic map was widely used to analyze the nonlinear phenomena in the switching converters [18-20]. In this work, the discrete time model of the Buck-Boost converter with switched-inductor is obtained by using the stroboscopic map.

Let $T$ be the switching period of the switch $S, i_{1, n}$ be the instantaneous value of $i_{L_{1}}$ at the beginning of $n$th cycle $t=n T$, and $u_{n}$ be the instantaneous value of $u_{C}$, then the solution of the state equation (7) can be given as

$$
\begin{gathered}
i_{L_{1}}(t)=i_{1, n}+\frac{E t}{L_{1}}, \\
u_{C}(t)=u_{n} e^{2 k t},
\end{gathered}
$$

where $k=-1 / 2 R C$.

Also, when the converter is working during "off" period, the solution of $i_{L_{1}}$ and $u_{C}$ in state equation (8) can be given as

$$
\begin{gathered}
i_{L_{1}}(t,)=e^{k t}\left(c_{1} \cos w t+c_{2} \sin w t\right), \\
u_{C}(t)=-\left(L_{1}+L_{2}\right) e^{k t} \\
\times\left[\left(k c_{1}+c_{2} w\right) \cos w t+\left(k c_{2}-c_{1} w\right) \sin w t\right],
\end{gathered}
$$

where

$$
\begin{aligned}
& R>\frac{1}{2} \sqrt{\frac{L_{1}+L_{2}}{C}}, \quad c_{1}=I_{\text {ref }} \\
& w=\sqrt{\frac{1}{\left(L_{1}+L_{2}\right) C}-(-k)^{2}},
\end{aligned}
$$

and

$$
c_{2}=\frac{1}{w}\left(\frac{-u_{n} e^{2 k t_{n}}}{L_{1}+L_{2}}-k c_{1}\right) .
$$

Based on the operating principle of the converter, the switching condition of the system can be defined as

$$
I_{\text {ref }}-i_{L_{1}}\left(t_{n}\right)=0 .
$$
by

According to (10) and (14), the on-time $t_{n}$ can be obtained

$$
t_{n}=L_{1} \times \frac{I_{\mathrm{ref}}-i_{1, n}}{E}
$$

If $t_{n} \geq T$ and the converter works in "on" state during the whole $n T \sim(n+1) T$, the values of $u_{n}$ and $i_{1, n}$ at the next clock instant are given by

$$
\begin{gathered}
i_{1, n+1}=i_{1, n}+\frac{E T}{L_{1}}, \\
u_{n+1}=u_{n} e^{2 k T} .
\end{gathered}
$$

If $t_{n}<T$, the converter works in "on" state during $n T \sim$ $\left(n T+t_{n}\right)$ and "off" state during $\left(n T+t_{n}\right) \sim(n+1) T$, the mapping are given by

$$
\begin{aligned}
& i_{1, n+1}=e^{k t_{m}}\left(c_{1} \cos w t_{m}+c_{2} \sin w t_{m}\right), \\
u_{n+1}= & -\left(L_{1}+L_{2}\right) e^{k t_{m}} \\
& \times\left[\left(k c_{1}+c_{2} w\right) \cos w t_{m}+\left(k c_{2}-c_{1} w\right) \sin w t_{m}\right],
\end{aligned}
$$

where $t_{m}$ is the off-time, and it can be calculated by

$$
t_{m}=T \times\left[1-\left(\frac{t_{n}}{T}\right) \bmod 1\right] .
$$

2.3. Voltage Conversion Ratio of the System. Let $U_{i}$ and $U_{o}$ be value of input voltage and output voltage at steady-state, respectively. Ignoring the resistance of the inductors and taking $L_{1}=L_{2}$ into consideration, the voltage drop on $L_{1}$ during the "on" period can be written as

$$
U_{L_{1}}=U_{i}
$$

The voltage drop on $L_{1}$ during the "off" period can be written as

$$
2 U_{L_{1}}=-U_{o}
$$

Thus, a voltage-second balance on $L_{1}$ can be written as

$$
D \cdot U_{i}+(1-D) \cdot\left(-\frac{1}{2} U_{o}\right)=0
$$

giving

$$
U_{o}=\frac{2 D}{1-D} U_{i}
$$

where $D$ is the duty cycle at steady-state.

Similarly, for a classical Buck-Boost converter, we can easily get that

$$
U_{o}=\frac{D}{1-D} U_{i}
$$

Comparing (22) with (23), it can be seen that the voltage conversion ratio of the new system is double that of the classical one.

\section{Analysis of Dynamic Characteristics}

For the power converters, many kinds of methods like phase portraits, bifurcation diagrams, and time-domain waveform 


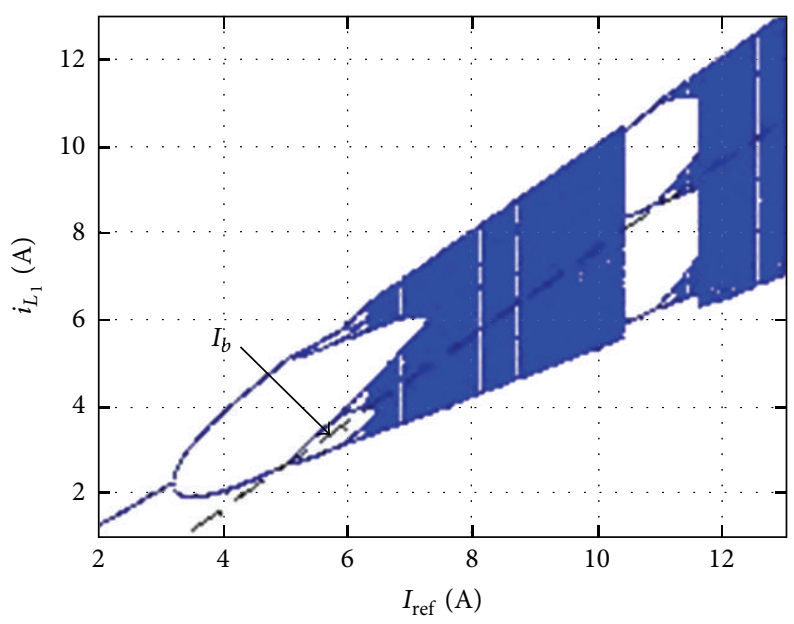

(a)

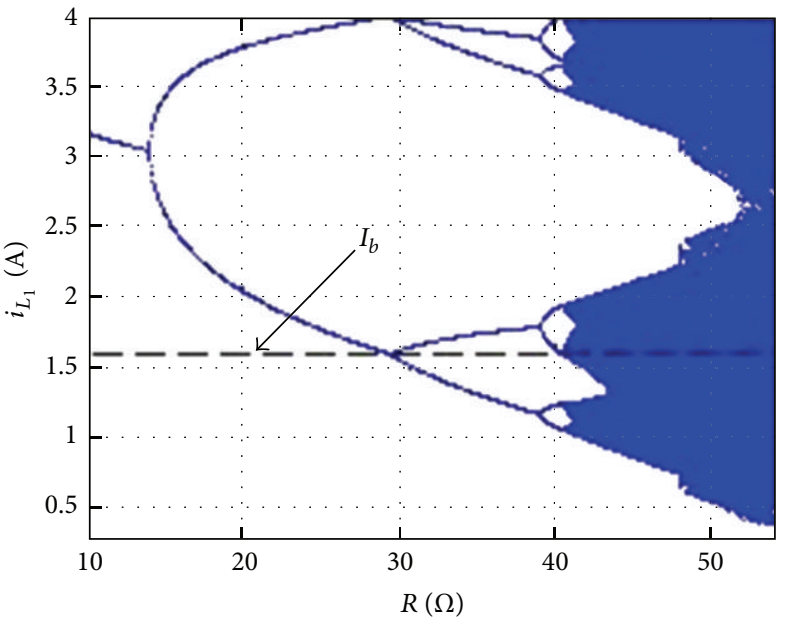

(b)

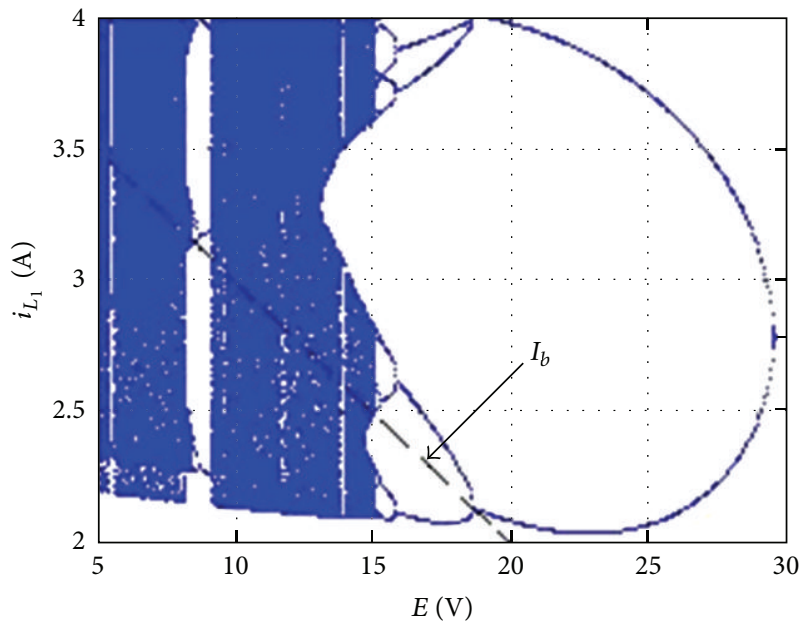

(c)

FIGURE 3: Bifurcation diagram with different circuit parameters. (a) $I_{\text {ref }}$ as parameter; (b) $R$ as parameter; (c) $E$ as parameter.

can be used to analyze the nonlinear phenomenon in the system. In this work, the bifurcation diagrams and Poincare sections are drawn based on the discrete iterated mapping model. Time-domain waveform, phase portraits, and power spectrum are obtained by building simulation module in Matlab/Simulink, which validates the theoretical analysis results.

3.1. Bifurcation Diagrams. It can be seen clearly from Figure 1 that there are six major parameters in the system: the reference current $I_{\text {ref }}$, inductance $L_{1}$ and $L_{2}$, load resistance $R$, input voltage $E$, and capacitance $C$. It is impossible to draw all the bifurcation diagrams for all possible parameter values and analyze them in detail in just one paper, so we present only a few typical cases. In order to facilitate the analysis of the complex dynamic behavior of the converter, (24) is given to calculate the borderline of $i_{L_{1}}$, which is denoted by $I_{b}$. Consider the following:

$$
I_{b}=I_{\text {ref }}-\frac{E T}{L_{1}}
$$

Let reference current $I_{\text {ref }}$ vary from $2 \mathrm{~A}$ to $13 \mathrm{~A}$ with a step of 0.02 A while other parameters are fixed at $E=24 \mathrm{~V}$, $L_{1}=1 \mathrm{mH}, L_{2}=1 \mathrm{mH}, C=4 \mu \mathrm{F}, R=20 \Omega$, and $f=$ $10 \mathrm{KHz}$. Figure 3(a) shows the bifurcation diagram with $I_{\text {ref }}$ as parameter, the dotted line in Figure 3(a) is the borderline of $i_{L_{1}}$ with the value of $I_{b}=I_{\text {ref }}-2.4$ according to (24). This diagram shows clearly period doubling bifurcations at $I_{\text {ref }}=3.25 \mathrm{~A}$. When $I_{\text {ref }}$ is equal to $5.25 \mathrm{~A}$, the period- 2 orbit collides with the borderline and turns to 4-periodic. Therefore, this period doubling phenomenon is not due to a standard pitchfork bifurcation, but because of a border collision bifurcation. The period- 4 orbit goes through a normal period doubling bifurcation at $I_{\text {ref }}=6 \mathrm{~A}$ and a period-8 orbit is created. Then, this orbit collides with the borderline again and turns into a chaotic orbit. Note that the chaotic state degenerate to 3-periodic when $I_{\text {ref }}$ is nearly equal to $11.5 \mathrm{~A}$. This special phenomenon is called tangent bifurcation, and the chaos that happened before tangent bifurcation is called intermittent chaos.

The bifurcation diagram with load resistance $R$ as parameter is shown in Figure 3(b). The load resistance $R$ is varied 


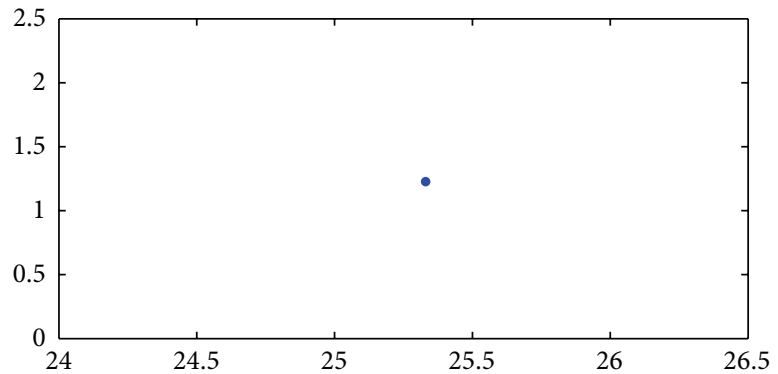

(a)

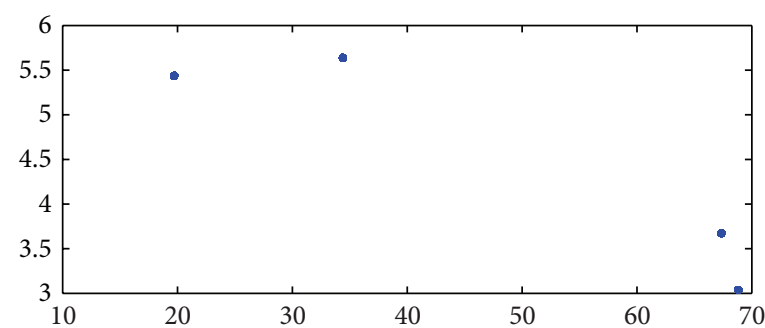

(c)

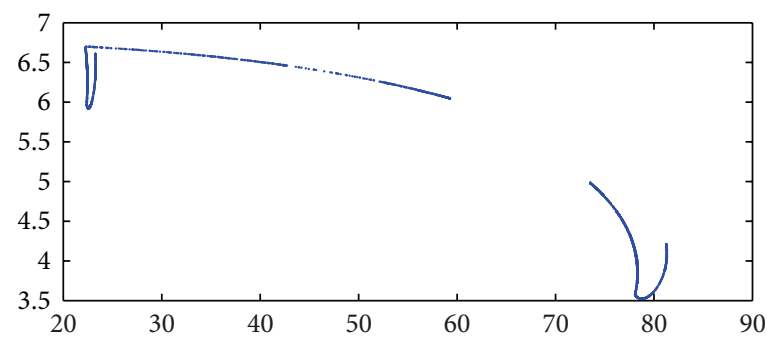

(e)

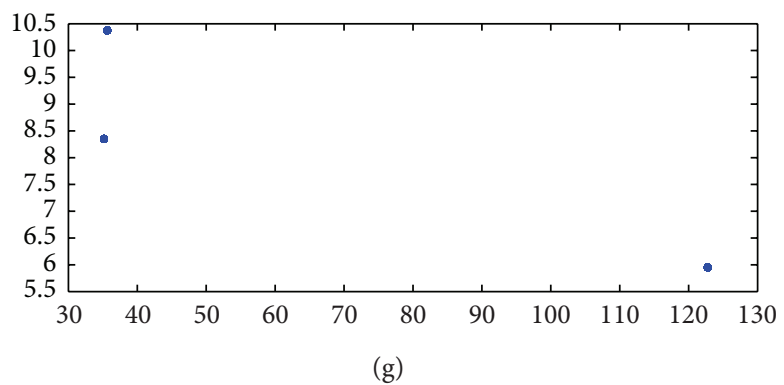

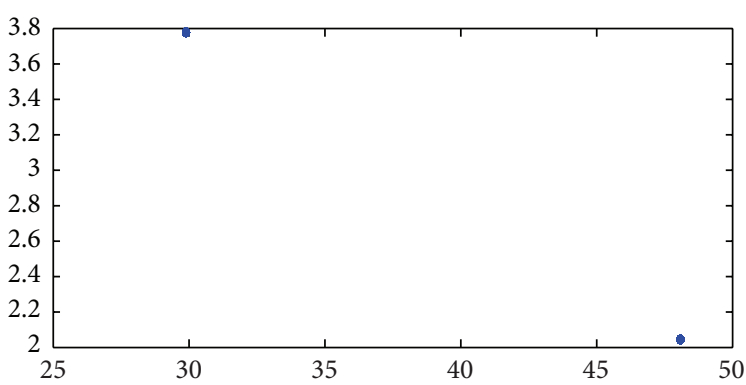

(b)

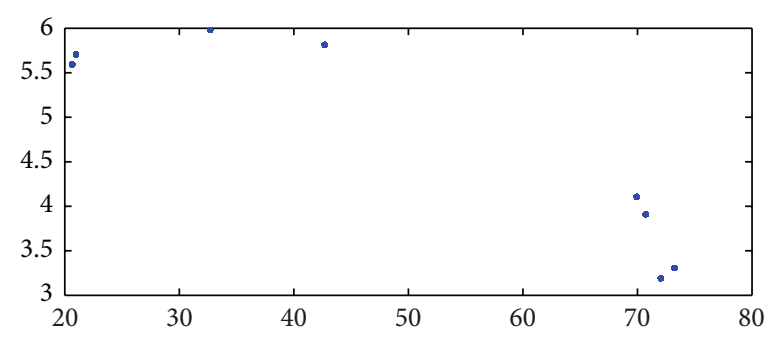

(d)

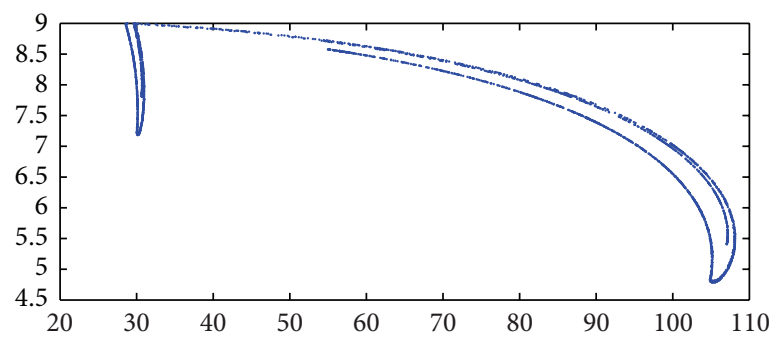

(f)

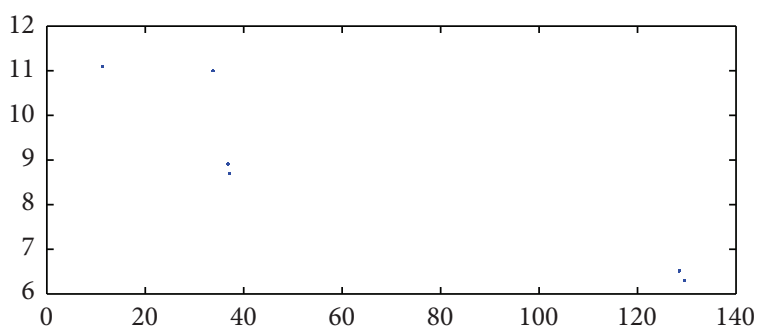

(h)

Figure 4: The Poincare sections under different value of reference current. (a) $I_{\text {ref }}=2 \mathrm{~A}$; (b) $I_{\text {ref }}=4 \mathrm{~A}$; (c) $I_{\text {ref }}=5.8 \mathrm{~A}$; (d) $I_{\text {ref }}=6.1 \mathrm{~A}$; (e) $I_{\text {ref }}=6.7 \mathrm{~A}$; (f) $I_{\text {ref }}=9 \mathrm{~A} ;(\mathrm{g}) I_{\text {ref }}=10.5 \mathrm{~A}$; (h) $I_{\text {ref }}=11.1 \mathrm{~A}$.

from $10 \Omega$ to $55 \Omega$ with a step of $0.05 \Omega$ while other parameters are fixed at $E=24 \mathrm{~V}, L_{1}=1 \mathrm{mH}, L_{2}=1 \mathrm{mH}, C=4 \mu \mathrm{F}, I_{\text {ref }}=$ $4 \mathrm{~A}$, and $f=10 \mathrm{KHz}$. Since the borderline is independent of $R$ according to (24), it is shown as a horizontal straight line with $I_{b}=1.6 \mathrm{~A}$ in this figure. And it can be seen clearly that the period doubling bifurcation occurs at $R=14 \Omega$, then the period- 2 orbit collides with the borderline at $R=30 \Omega$ and a stable period- 4 orbit is generated. The period- 4 orbit goes through a normal period doubling bifurcation at $R=$ $39 \Omega$ and a period- 8 orbit is created, which collides with the borderline at $R=40.5 \Omega$ and finally turns into a chaotic orbit. It can be noted that there are no periodic windows within the chaotic region.
The bifurcation diagram with input voltage $E$ as parameter is shown in Figure 3(c). The input voltage $E$ is varied from $5 \mathrm{~V}$ to $30 \mathrm{~V}$ with a step of $0.05 \mathrm{~V}$ while other parameters are fixed at $R=20 \Omega, L_{1}=1 \mathrm{mH}, L_{2}=1 \mathrm{mH}, C=4 \mu \mathrm{F}$, $I_{\text {ref }}=4 \mathrm{~A}$, and $f=10 \mathrm{KHz}$. Thus, the borderline of $i_{L_{1}}$ can be obtained by $I_{b}=4-0.1 E$ according to (24). It can be seen clearly from Figure 3(c) that with the change of the input voltage $E$, its route to chaos is similar with Figure 3(a), including period-doubling bifurcation, border collision bifurcation, more than once tangent bifurcation, and intermittent chaos. At the same time, the diagram reveals that the bifurcation occurs with the decrease of $E$, which is opposite to the change of $I_{\text {ref }}$ and $R$. And it is worth noting 


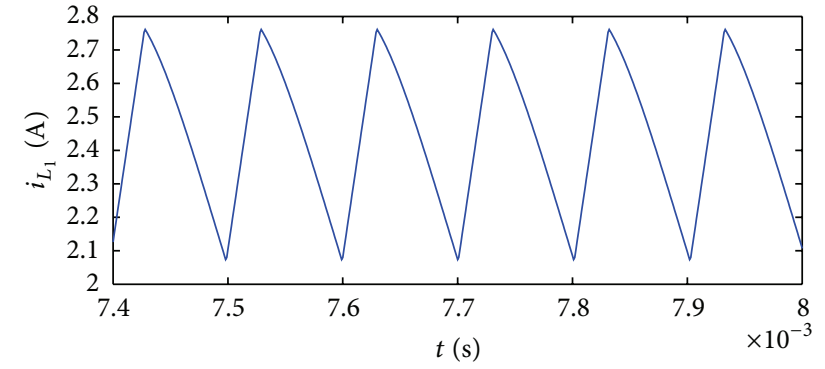

(a)

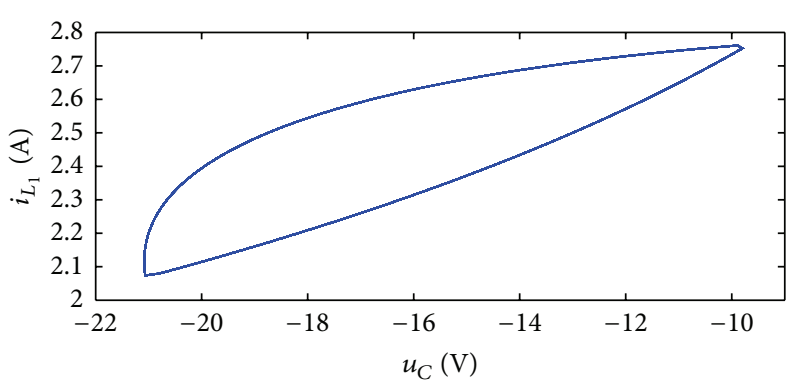

(b)

FIgURE 5: The time-domain waveform and phase portrait when $R=10 \Omega$. (a) Time-domain waveform; (b) phase portrait.

that the 3-periodic window is the most obvious periodic window in the intermittent chaotic region.

3.2. Poincare Sections. Poincare sections can be an effective method to analyze the dynamic characteristics of nonlinear systems. According to nonlinear dynamics theory, the performance of the system can be judged by observing the number of the cutoff points on the Poincare sections. One point or a few discrete points indicates that the converter is working in periodical state, while the cutoff point with a fractal structure reveals a chaotic behavior.

To prove that when the reference current $I_{\text {ref }}$ changes from $2 \mathrm{~A}$ to $13 \mathrm{~A}$, the converter exists; the nonlinear phenomena shown is in Figure 3(a), Figure 4 gives the Poincare sections under different value of $I_{\text {ref }}$.

It can be seen clearly from Figures $4(\mathrm{a})-4(\mathrm{~d})$ that the points on the Poincare sections are discrete when $I_{\text {ref }}$ is equal to $2 \mathrm{~A}, 4 \mathrm{~A}, 5.8 \mathrm{~A}$, and $6.1 \mathrm{~A}$, respectively. The converter is working in period-1, period-2, period-4, and period-8 successively according to the number of the points. For Figures 4(e) and 4(f), the cutoff points act as a banded structure, indicating that the converter is operating in chaotic state when $I_{\text {ref }}$ is equal to 6.7 A or 9 A. Figures $4(\mathrm{~g})$ and $4(\mathrm{~h})$ show that there is a 3-periodic window in the intermittent chaotic region, and the 3-periodic orbit turns to period6 before it comes into chaos. The above consequences are coinciding with the results shown in Figure 3(a). Therefore, the Poincare sections further prove the correctness of the discrete-time mapping model and reflect the operating state of the converter under different value of $I_{\text {ref }}$ more vividly.

3.3. Time-Domain Waveform and Phase Portraits. The bifurcation diagram in Figure 3(b) shows that the converter goes through period-1, period-2, period-4, and period- 8 and finally turns into intermittent chaos when the load resistance increases from $10 \Omega$ to $55 \Omega$. In order to prove the existence of the above phenomena, we set up a simulation model by Matlab/Simulink in this section according to Figure 1, and we select different value of $R$ in this simulation model; the corresponding time-domain waveforms and phase portraits are shown inFigures 5, 6, 7, 8 and 9,

From Figures $5-8$, we can easily find that when the load resistance is equal to $10 \Omega, 20 \Omega, 35 \Omega$, and $39.5 \Omega$, the state of the system is corresponding to period-1, period-2, period-4, and period-8, respectively. Note that the phase portrait in Figure $8(\mathrm{~b})$ cannot reflect the periodicity of the converter clearly, so we give the enlarged view of it in Figure 8(c) in order to illustrate the number of the cycles better.

The time-domain waveform and phase portrait when the load resistance is equal to $55 \Omega$ are shown in Figure 9. It can be observed that the time-domain waveform appears to be random, and there is a strange attractor in the phase portrait, which indicates that the converter is working in chaotic state.

According to the time-domain waveforms and phase portraits from Figure 5 to Figure 9, it can be seen clearly that the conclusions obtained by Matlab/Simulink are in good agreement with the results got by bifurcation diagram, which confirms the validity of the theoretical analysis better.

3.4. Power Spectrum. The power spectrum is also an effective way to analyze the stability of the circuit. When the circuit operates in the cycle $n$, the peak of the waveform will only appear when the frequency is equal to $i / n$ times of the operating frequency ( $i$ is a positive integer). When the circuit is in a chaotic state, the power spectrum manifests as a continuous spectrum, which contains a peak corresponding to the periodic motion. Figures $10(\mathrm{a})-10$ (d) clearly reveal that when the load resistance is equal to $10 \Omega, 20 \Omega, 35 \Omega$, and $55 \Omega$, the converter works in period-1, period-2, period4 , and chaotic state, respectively. The results are in good agreement with the previous analysis, which further verify the correctness of the results obtained by theoretical analysis.

\section{Conclusion}

Inserting the switched-inductor structure into traditional Buck-Boost converter can increase its voltage conversion ratio significantly. However, it will lead to a conversion of the inductors from series connection to parallel connection, which makes it easier for the system to be affected by the circuit parameters. In this paper, the nonlinear behaviors of the Buck-Boost converter with switched-inductor structure have been studied systematically. The discrete iterated mapping model under CCM is established. The effects of circuit parameters on system performance are analyzed using bifurcation diagrams and Poincare sections. Then, the corresponding 


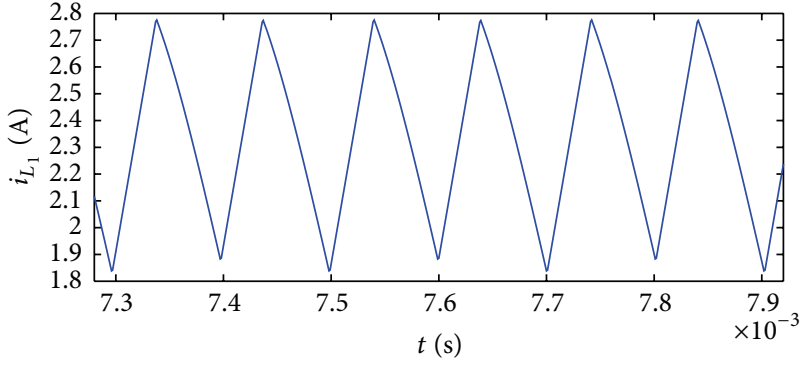

(a)

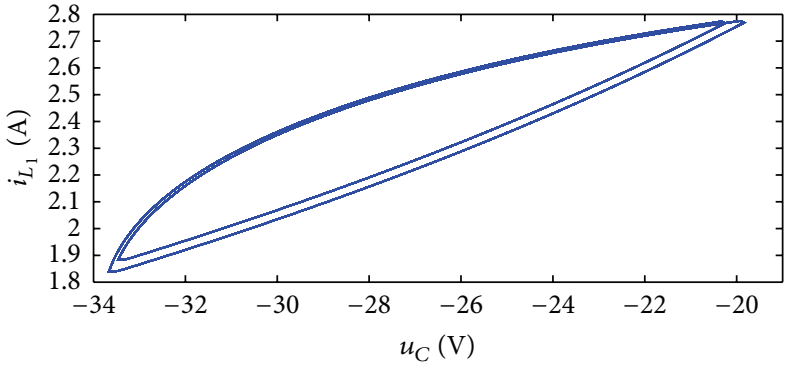

(b)

FIgURE 6: The time-domain waveform and phase portrait when $R=20 \Omega$. (a) Time-domain waveform; (b) phase portrait.

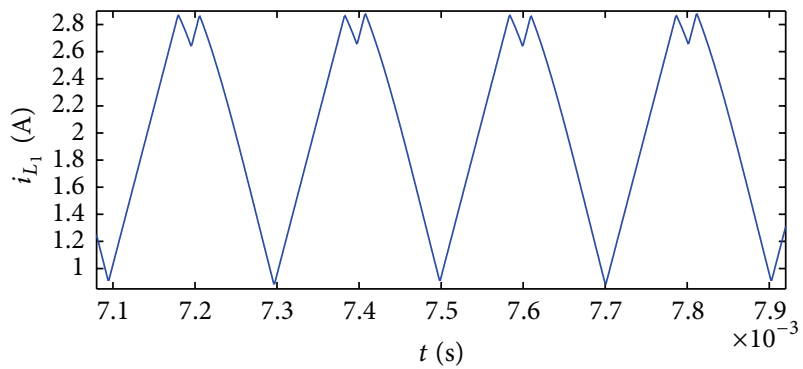

(a)

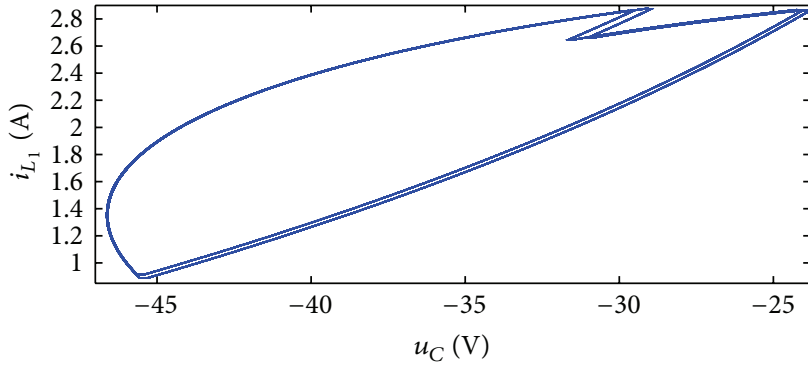

(b)

FIgURE 7: The time-domain waveform and phase portrait when $R=35 \Omega$. (a) Time-domain waveform; (b) phase portrait.

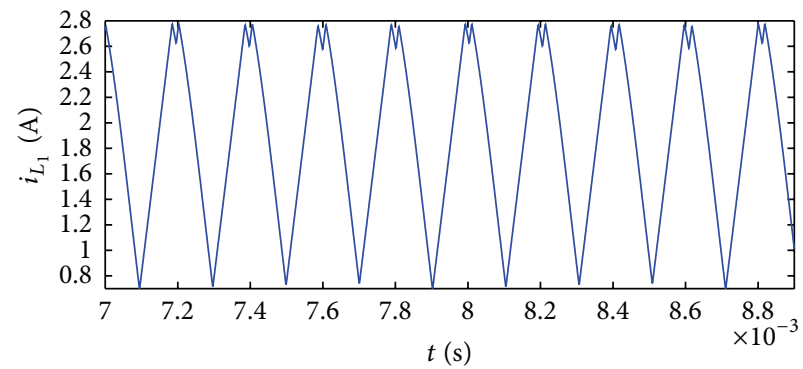

(a)

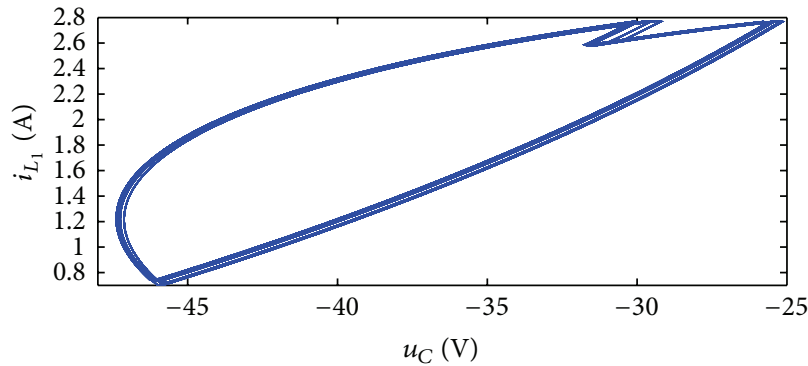

(b)

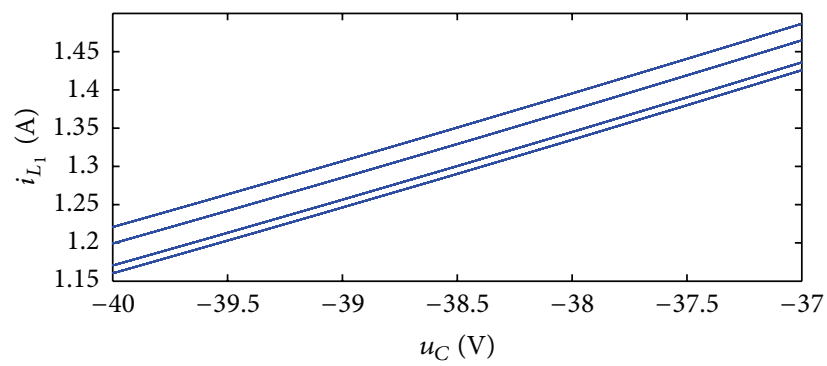

(c)

FIGURE 8: The time-domain waveform and phase portrait when $R=39.5 \Omega$. (a) Time-domain waveform; (b) phase portrait; (c) the partially enlarged view of phase portrait.

time-domain waveforms, phase portraits, and power spectrum are obtained by Matlab/Simulink. The research results show that various kinds of nonlinear phenomena are easy to come in this new converter, including period-doubling bifurcation, border collision bifurcation, tangent bifurcation, and intermittent chaos. The results between the theoretical analysis and the simulation are in general agreement with each other. According to the analysis above, the BuckBoost converter with switched-inductor structure belongs to strongly nonlinear system, and its performance can be easily 


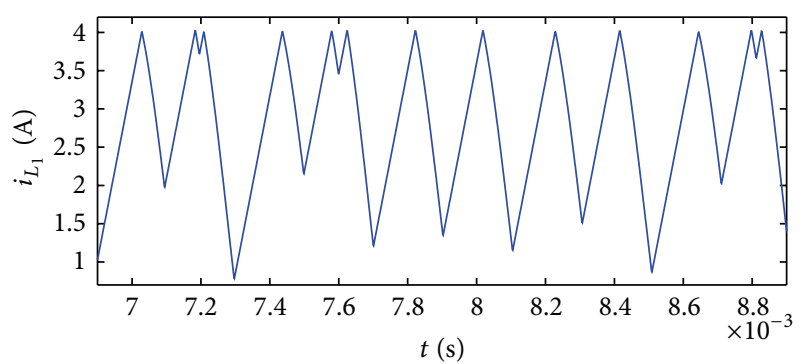

(a)

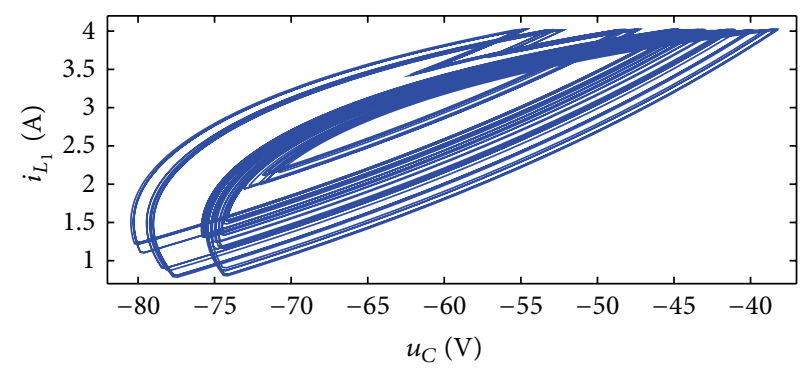

(b)

FIgURE 9: The time-domain waveform and phase portrait when $R=55 \Omega$. (a) Time-domain waveform; (b) phase portrait.

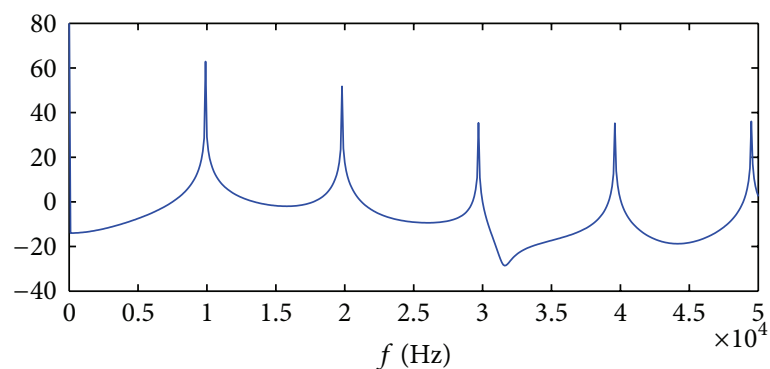

(a)

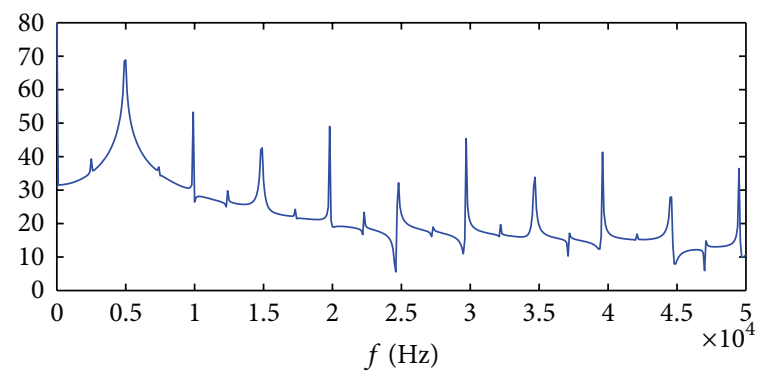

(c)

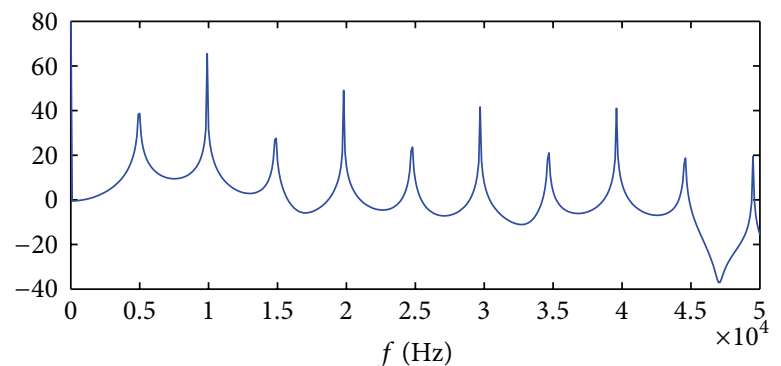

(b)

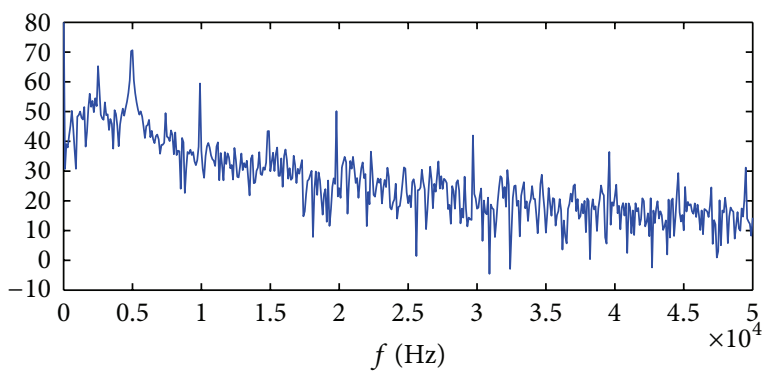

(d)

FigURE 10: The power spectrum under different value of load resistance. (a) $R=10 \Omega$; (b) $R=20 \Omega$; (c) $R=35 \Omega$; (d) $R=55 \Omega$.

affected when the circuit parameters are varied. Therefore, the parameters should be chosen appropriately according to the research results. The analysis methods and research findings will possess an important reference value to engineering design and performance analysis.

\section{Acknowledgments}

This work is supported by the National Natural Science Foundation of China (no. 51107016), the National Key Basic Research Program of China (973 Program) under Grant (2013CB035605), and the Postdoctoral scienceresearch developmental foundation of Heilongjiang province (no. LHB-Q12086).

\section{References}

[1] B. Axelrod, Y. Berkovich, and A. Ioinovici, "Switched-capacitor/ switched-inductor structures for getting transformerless hybrid
DC-DC PWM converters," IEEE Transactions on Circuits and Systems I, vol. 55, no. 2, pp. 687-696, 2008.

[2] B. Axelrod, Y. Berkovich, and A. Ioinovici, "Switched-capacitor scyswitchedinductor (SL) structures for getting hybrid stepdown Cuk/Sepic/Zeta converters," in Proceedings of the IEEE International Symposium on Circuits and Systems (ISCAS '06), pp. 5063-5066, May 2006.

[3] C. K. Tse and M. Di Bernardo, "Complex behavior in switching power converters," Proceedings of the IEEE, vol. 90, no. 5, pp. 768-781, 2002.

[4] A. El Aroudi and R. Leyva, "Quasi-periodic route to chaos in a PWM voltage-controlled dc-dc boost converter," IEEE Transactions on Circuits and Systems I, vol. 48, no. 8, pp. 967978, 2001.

[5] S. K. Mazumder, A. H. Nayfeh, and D. Boroyevich, "Theoretical and experimental investigation of the fast- and slow-scale instabilities of a DC-DC converter," IEEE Transactions on Power Electronics, vol. 16, no. 2, pp. 201-216, 2001.

[6] M. Debbat, A. El Aroudi, R. Giral, and L. Martínez-Salamero, "Hopf bifurcation in PWM controlled asymmetrical interleaved 
dual boost DC-DC converter," in Proceedings of the IEEE International Conference on Industrial Technology, vol. 2, no. 2, pp. 860-865, December 2003.

[7] D. Dai, C. K. Tse, and X. Ma, "Symbolic analysis of switching systems: application to bifurcation analysis of DC/DC switching converters," IEEE Transactions on Circuits and Systems I, vol. 52, no. 8, pp. 1632-1643, 2005.

[8] H. H. C. Iu and C. K. Tse, "A study of synchronization in chaotic autonomous ćuk dc/dc converters," IEEE Transactions on Circuits and Systems I, vol. 47, no. 6, pp. 913-918, 2000.

[9] C. K. Tse, Y. M. Lai, and H. H. C. Lu, "Hopf bifurcation and chaos in a free-running current-controlled Cuk switching regulator," IEEE Transactions on Circuits and Systems I, vol. 47, no. 4, pp. 448-457, 2000.

[10] A. Kavitha and G. Uma, "Control of chaos in SEPIC DC-DC converter," International Journal of Control, Automation and Systems, vol. 8, no. 6, pp. 1320-1329, 2010.

[11] Y. Ma, C. K. Tse, T. Kousaka, and H. Kawakami, "Connecting border collision with saddle-node bifurcation in switched dynamical systems," IEEE Transactions on Circuits and Systems II, vol. 52, no. 9, pp. 581-585, 2005.

[12] A. M. Harb, I. Batarseh, L. M. Mili, and M. A. Zohdy, "Bifurcation and chaos theory in electrical power systems: analysis and control," Mathematical Problems in Engineering, vol. 2012, Article ID 573910, 2 pages, 2012.

[13] A. El Aroudi, E. Rodríguez, R. Leyva, and E. Alarcón, "A design-oriented combined approach for bifurcation prediction in switched-mode power converters," IEEE Transactions on Circuits and Systems II, vol. 57, no. 3, pp. 218-222, 2010.

[14] B. Basak and S. Parui, "Exploration of bifurcation and chaos in buck converter supplied from a rectifier," IEEE Transactions on Power Electronics, vol. 25, no. 6, pp. 1556-1564, 2010.

[15] J. Wu, M. Liu, and P. Yang, "Study of bifurcation and chaos in the current-mode controlled Buck-Boost DC-DC converter (I)modeling and simulation," Control Theory and Applications, vol. 19, no. 3, pp. 387-394, 2002.

[16] J. Wu, M. Liu, and P. Yang, "Study of bifurcation and chaos in the current-mode controlled Buck-Boost DC-DC converter (II)-numerical analysis and experiment," Control Theory and Applications, vol. 19, no. 3, pp. 395-401, 2002.

[17] B. Bao, J. Xu, and Z. Liu, "Mode shift and stability control of a current mode controlled buck-boost converter operating in discontinuous conduction mode with ramp compensation," Chinese Physics B, vol. 18, no. 11, pp. 4742-4747, 2009.

[18] M. D. Bernardo and F. Vasca, "Discrete-time maps for the analysis of bifurcations and chaos in DC/DC converters," IEEE Transactions on Circuits and Systems I, vol. 47, no. 2, pp. 130-143, 2000.

[19] S. Banerjee and K. Chakrabarty, "Nonlinear modeling and bifurcations in the boost converter," IEEE Transactions on Power Electronics, vol. 13, no. 2, pp. 252-260, 1998.

[20] C. K. Tse, "Flip bifurcation and chaos in three-state boost switching regulators," IEEE Transactions on Circuits and Systems I, vol. 41, no. 1, pp. 16-23, 1994. 


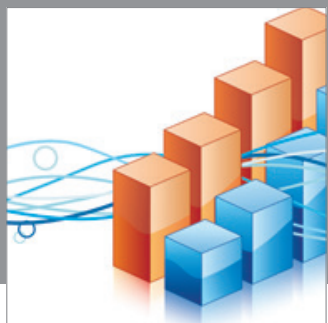

Advances in

Operations Research

mansans

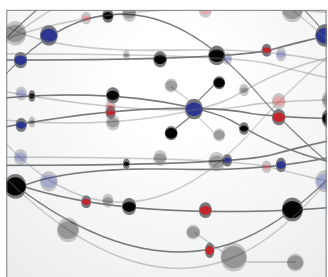

The Scientific World Journal
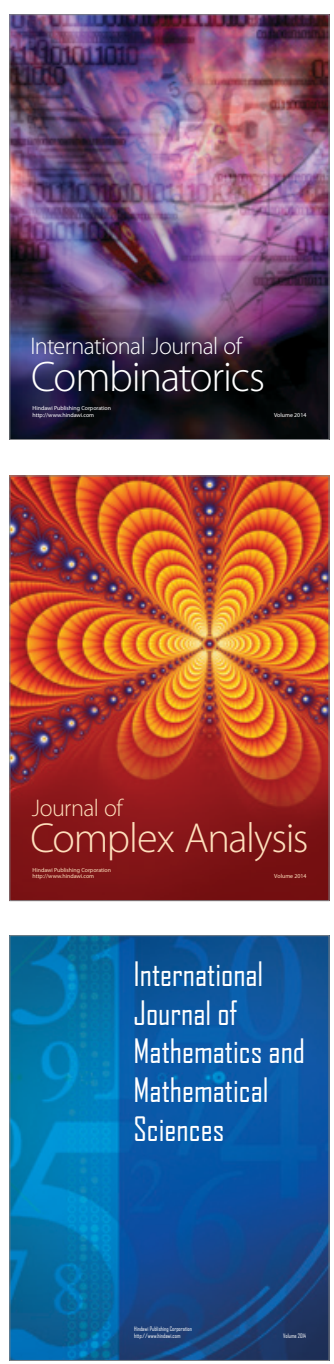
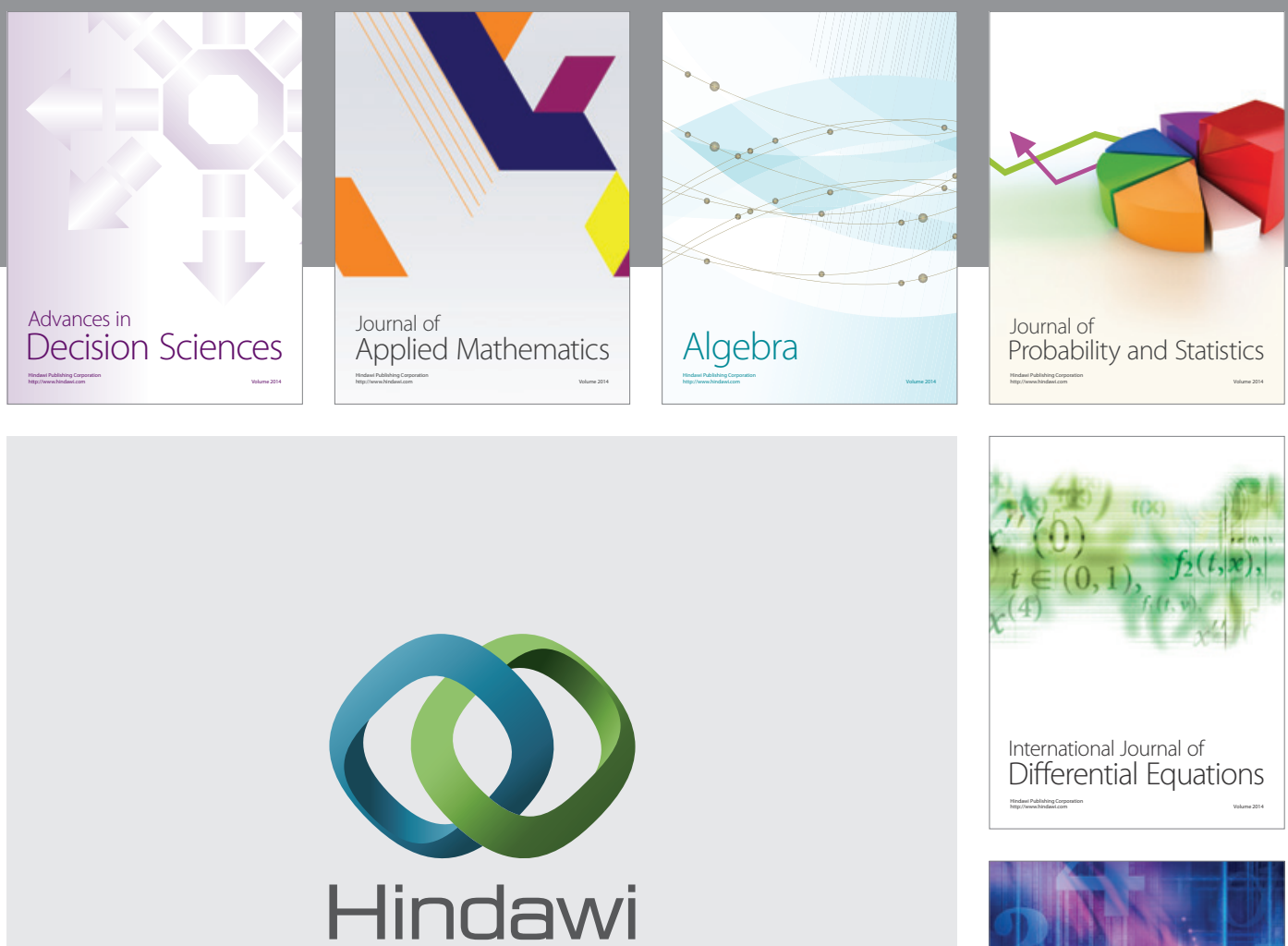

Submit your manuscripts at http://www.hindawi.com
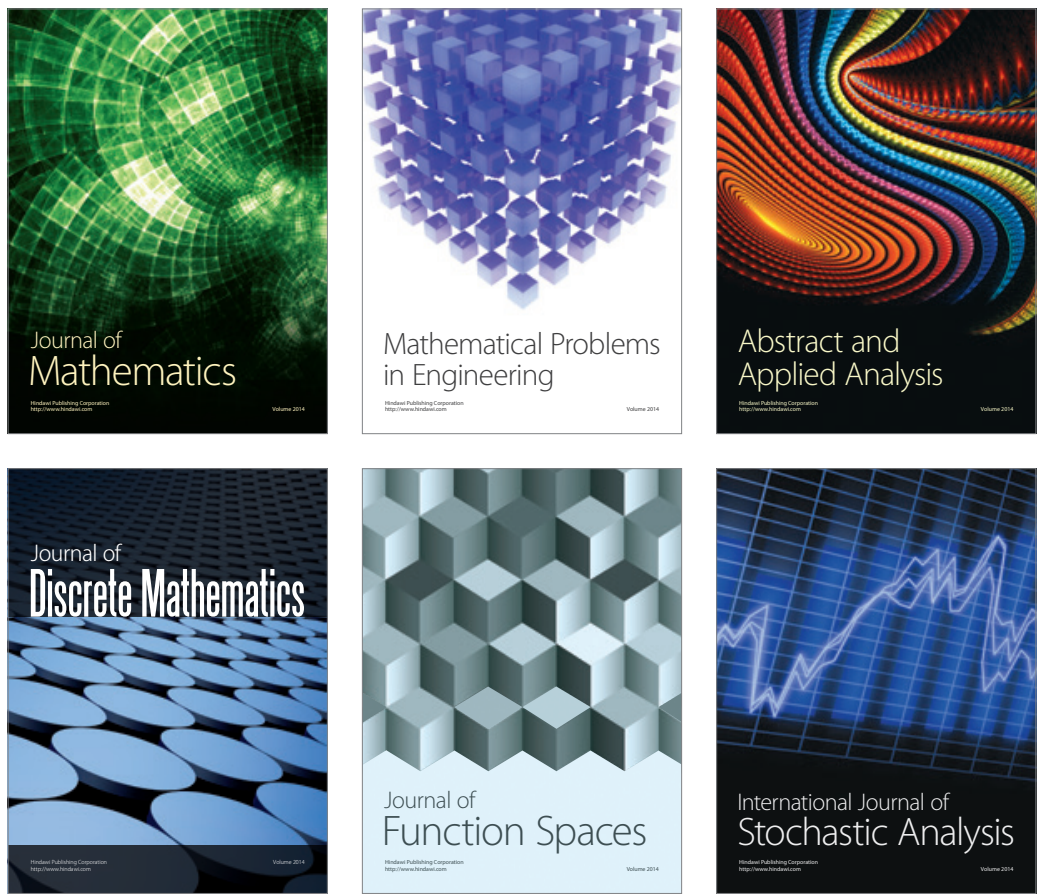

Journal of

Function Spaces

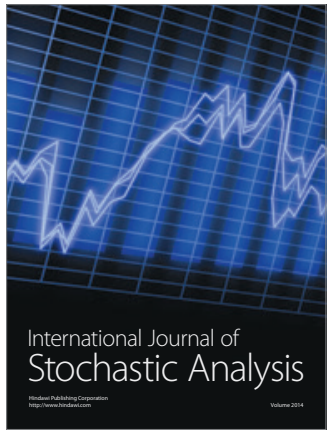

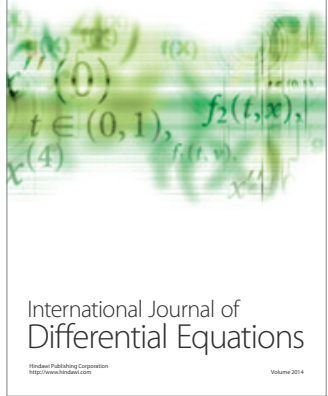
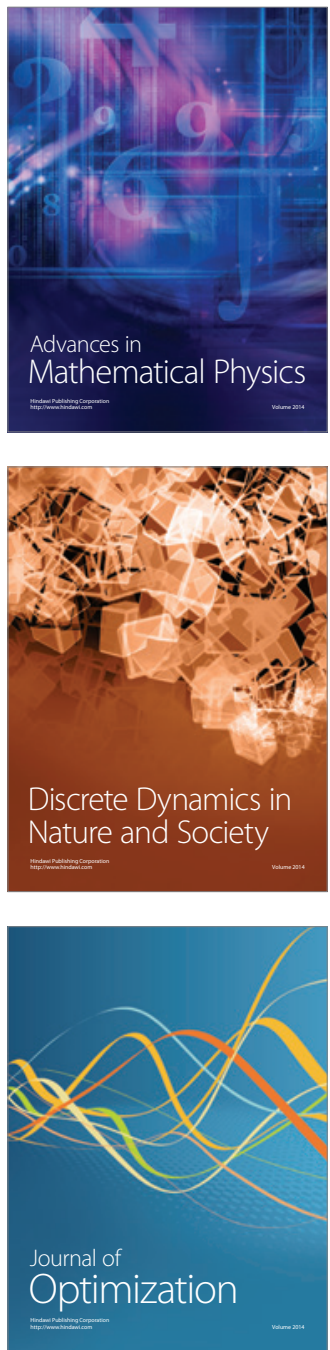